\title{
Holter Monitoring
}

National Cancer Institute

\section{Source}

National Cancer Institute. Holter Monitoring. NCI Thesaurus. Code C38064.

An ambulatory electrocardiog raphy technique that records a continuous

electrocardiographic rhythm pattern for 24 hours or more to detect heart arrhythmias. 